\title{
Identification of Emerging Human Mastitis Pathogens by MALDI-TOF and Assessment of Their Antibiotic Resistance Patterns
}

OPEN ACCESS

Edited by:

M. Pilar Francino,

Public Health - FISABIO, Spain

Reviewed by:

Luis Carlos Guimarães, Federal University of Pará, Brazil

Chris Whitehouse,

United States Food and Drug

Administration, United States

*Correspondence:

María Marín

m/marin@ucm.es

Juan M. Rodríguez

jmrodrig@ucm.es

Specialty section: This article was submitted to Antimicrobials, Resistance and Chemotherapy,

a section of the journal Frontiers in Microbiology

Received: 03 May 2017 Accepted: 23 June 2017

Published: 12 July 2017

Citation:

Marín M, Arroyo $R$ Espinosa-Martos I, Fernández $L$ and Rodríguez JM (2017) Identification of Emerging Human Mastitis

Pathogens by MALDI-TOF and Assessment of Their Antibiotic

Resistance Patterns.

Front. Microbiol. 8:1258.

doi: 10.3389/fmicb.2017.01258

\section{María Marín ${ }^{1 *}$, Rebeca Arroyo², Irene Espinosa-Martos², Leónides Fernández ${ }^{1}$ and Juan M. Rodríguez ${ }^{1 *}$}

${ }^{1}$ Departamento de Nutrición, Bromatología y Tecnología de los Alimentos, Universidad Complutense de Madrid, Madrid, Spain, ${ }^{2}$ Probisearch, Madrid, Spain

Lactational mastitis constitutes one of the main causes of undesired weaning, depriving the mother-infant pair from the benefits of breastfeeding; therefore, this condition should be considered a relevant public health issue. The role of specific microorganisms remains unclear since human milk cultures and antibiotic susceptibility testing (AST) are not routinely performed, despite the fact that this would be key to ensure an early and effective diagnosis and treatment. The objective of this study was to describe the culturable microbial diversity in 647 milk samples from breastfeeding women with clinical symptoms of mastitis by Matrix-Assisted Laser Desorption Ionization Time-of-Flight Mass Spectrometry (MALDI-TOF) VITEK MS technology and to analyze the antimicrobial susceptibility profiles of a collection of isolates from these samples by the VITEK 2 AST system. Staphylococcus epidermidis was the most common species isolated from mastitis samples (87.6\%), while Staphylococcus aureus was detected in 22.1\%. Streptococci constituted the second (68.6\%) most prevalent bacterial group, with Streptococcus mitis/oralis, Streptococcus salivarius, and Streptococcus parasanguinis detected with frequencies of 40.8, 36.8, and $14.4 \%$, respectively. The antibiotic susceptibility profiles of 642 staphylococcal isolates indicated a remarkable resistance to benzylpenicillin (88.3\%) and erythromycin (67.3\%) with differences between species. A high percentage of Staphylococcus isolates were resistant to at least one antibiotic (Staphylococcus hominis, 100\%; S. epidermidis, 98.2\%; S. aureus, 92.9\%; Staphylococcus lugdunensis, 90.5\%) and the percentage of multidrug-resistance (MDR) isolates was noticeable (S. hominis, $81 \%$; S. epidermidis, 64.4\%; S. aureus, 11.5\%; S. lugdunensis, 10.5\%). In relation to streptococcal isolates $(n=524)$, AST revealed high or moderate percentages of resistance to erythromycin (68.7\%), benzylpenicillin (63.7\%), ampicillin (51.5\%), and tetracycline (30.8\%). Antibiotic resistance to at least one antibiotic was detected in $97.6 \%$ of $S$. parasanguinis, $92.6 \%$ of S. salivarius, $83.3 \%$ of S. mitis/oralis, and $72.4 \%$ of Streptococcus vestibularis isolates. A significant number of MDR streptococcal isolates was also found (S. parasanguinis, 51.2\%; S. salivarius, 39.3\%; S. mitis/oralis, 34.6\%; and S. vestibularis, 19\%). The results 
highlight the important role of coagulase-negative staphylococci and streptococci as human mastitis-causing agents. Moreover, the high rates of antimicrobial resistance among these microorganisms must be contemplated as an issue of clinical relevance in relation to treatment options.

Keywords: human mastitis, breastfeeding, mastitis pathogens, antibiotic resistance, MALDI-TOF, antimicrobial susceptibility testing

\section{INTRODUCTION}

Infectious mastitis is a common condition that affects up to $33 \%$ of women during lactation and constitutes one of the main causes of undesired weaning (WHO, 2000; Foxman et al., 2002; Scott et al., 2008; Amir and Academy of Breastfeeding Medicine Protocol Committee, 2014), depriving the motherinfant pair from the health benefits of breastfeeding (U.S. Department of Health and Human Services, 2011). This pathological condition is characterized by a dysbiosis process in the mammary gland, leading to an overgrowth of certain bacterial species present in human milk (Delgado et al., 2008; Contreras and Rodríguez, 2011; Fernández et al., 2013). However, the role of specific microorganisms still remains unclear since human milk cultures and antibiotic susceptibility testing (AST) are not routinely performed (Scott et al., 2008; Barlow, 2011; Contreras and Rodríguez, 2011). The lack of this information prompts the empiric use of broadspectrum antibiotics giving rise to a frequent therapeutic failure (Fernández and Rodríguez, 2014). In this sense, a rapid and reliable identification of the microorganisms involved in mastitis together with the determination of their antimicrobial susceptibility profile is relevant to ensure an early and effective diagnosis and treatment, leading to a decrease in antimicrobial resistance rates and lower treatment costs.

Until recently, microbial identification in clinical microbiology laboratories has mostly relied on conventional methods that involve culturing, morphologic phenotyping, and biochemical testing. These procedures often require large amounts of biological materials, are time-consuming and the species assignment may be imprecise. Molecular identification methods mainly based on the sequencing of housekeeping genes, such as $16 \mathrm{~S}$ rRNA, are more discriminating than phenotypic tests, but also more complicated and expensive; therefore, they are not ideal for application on a routine basis in clinical laboratories. In the last years, the application of matrix-assisted laser desorption ionization-time of flight mass spectrometry (MALDI-TOF MS) technology has emerged as an accurate, rapid and cost-effective tool for the identification of clinically relevant microorganisms in large laboratories, providing a valuable alternative to phenotypic and molecular methods (Bizzini et al., 2011; Nomura, 2015; Singhal et al., 2015). This approach has been used successfully to identify bovine mastitis pathogens (Tomazi et al., 2014; Schmidt et al., 2015); however, it has been scarcely applied to date for the identification of microorganisms causing human mastitis.

Different commercial systems have been developed for AST due to their ease of use and interpretation, speed and cost-effectiveness in relation to the laborious standard methods of broth microdilution and disk diffusion. Among them, VITEK 2 (bioMérieux, Marcy l'Étoile, France) is an automated AST system that provides determinations of the minimum inhibitory concentration (MIC) within a few hours after isolation and integrates an expert system (Advanced Expert System) for biological validation of susceptibility results and therapeutic interpretation (Winstanley and Courvalin, 2011).

In this context, the objective of this study was to describe the microbial diversity in 647 milk samples from breastfeeding women with clinical symptoms of mastitis by MALDI-TOF VITEK MS technology and to analyze the antimicrobial susceptibility profiles of a collection of 1,180 mastitis isolates obtained from these samples by the VITEK 2 AST system.

\section{MATERIALS AND METHODS}

\section{Isolation of Bacteria from Milk Samples}

Milk samples ( $n=647)$ were obtained from women with clinical symptoms of infectious mastitis. All cases included either both local (breast redness, pain, engorgement, and reduced milk secretion) and systemic symptoms (fever or flulike symptoms) or only local symptoms. Written informed consent to the protocol (reference 10/017E) approved by the Ethical Committee of Hospital Clínico San Carlos (Madrid, Spain) was obtained from the women that provided the biological samples of this study. In the case of women with previous antibiotic therapy (33\%, based on patient self-report of antibiotic use), samples were collected at least 2 weeks after finishing the treatment. Milk samples for microbial analysis were collected following the protocol proposed by Delgado et al. (2015). Briefly, nipple and mammary areola were cleaned with soap and water and a milk sample $(\sim 1 \mathrm{ml})$ was collected aseptically in a sterile tube by manual expression after discarding the first drops. The use of milk pumps for sample collection was absolutely discouraged to avoid contamination. Milk samples were plated onto ready-to-use Columbia nalidixic acid agar plates (bioMérieux, Madrid, Spain) and incubated at $37^{\circ} \mathrm{C}$ for $48 \mathrm{~h}$. In order to reach a positive diagnosis of mastitis, the presence of different bacterial morphotypes was investigated and the following microbiological criteria were established: coagulasenegative staphylococci, viridans group streptococci (VGS), and other species > 1,000 CFU/ml; Staphylococcus aureus and Corynebacterium spp. $>150 \mathrm{CFU} / \mathrm{ml}$, since the latter could lead to mastitis at much lower concentrations. 


\section{Identification by MALDI-TOF Mass Spectrometry}

A total of 2,323 isolates from the mastitis milk samples were identified by MALDI-TOF using a VITEK MS instrument (bioMérieux, Marcy l'Etoile, France) in the facilities of Probisearch (Tres Cantos, Madrid, Spain). Briefly, a portion of a bacterial colony $(\sim 1 \mu \mathrm{L})$ was spotted onto a MALDI sample plate, overlaid with $1 \mu \mathrm{L}$ of a saturated solution of $\alpha$-cyano-4hydroxycinnamic acid in acetonitrile (28\%), and then allowed to dry at room temperature. For each isolate, a mean spectrum was constructed with at least $50 \mathrm{~m} / z$ spectra profiles and used for the identification by comparison with the spectra contained in the Myla database (bioMérieux). Identification was defined as a $99-100 \%$ match to the species-specific $\mathrm{m} / \mathrm{z}$ profiles in the database.

\section{Antimicrobial Susceptibility Testing}

A total of 1,180 staphylococcal, streptococcal and enterococcal isolates, previously identified at species level by MALDI-TOF, were subjected to AST with the VITEK 2 technology (bioMérieux). The Staphylococcus isolates $(n=642)$ belonged to Staphylococcus aureus $(n=140)$, Staphylococcus epidermidis ( $n=435)$, Staphylococcus hominis $(n=21)$, Staphylococcus lugdunensis $(n=21)$, and other minority species $(n=25)$. The Streptococcus isolates $(n=524)$ belonged to Streptococcus mitis/oralis $(n=215)$, Streptococcus parasanguinis $(n=82)$, Streptococcus salivarius $(n=162)$, Streptococcus vestibularis $(n=29)$ and other minority species $(n=36)$. MALDI-TOF MS Vitek instrument was unable to discriminate between the species $S$. mitis and $S$. oralis. All enterococal isolates $(n=14)$ belonged to Enterococcus faecalis. The isolates were subcultured on Columbia nalidixic acid agar plates and incubated at $37^{\circ} \mathrm{C}$ for $48 \mathrm{~h}$. Colonies were resuspended in $0.45 \% \mathrm{NaCl}$ solution and the bacterial suspension was adjusted to the turbidity of 0.5-0.6 McFarland standard with an ATB 1550 densitometer (bioMérieux) and properly diluted to inoculate the test cards for VITEK 2 according to the manufacturer's instructions. The following test cards (bioMérieux) were used: AST-P626 (Staphylococcus spp.), AST-ST01 (VGS and Streptococcus pyogenes) and AST-P589 (Enterococcus spp. and Streptococcus agalactiae). Antimicrobial concentration ranges and quality control strains of these cards were established according to the Clinical and Laboratory Standards Institute (CLSI) recommendations (Clinical and Laboratory Standards Institute [CLSI], 2013). The resulting MIC values were categorized into clinical categories of susceptible, intermediate or resistant following the interpretive criteria of the VITEK 2 expert system based on the CLSI guidelines (Clinical and Laboratory Standards Institute [CLSI], 2013). Isolates with MIC in the intermediate range were classified as resistant for data analysis. Resistance to, at least, one antimicrobial and multidrugresistance (MDR) were calculated for the main Staphylococcus and Streptococcus species isolated from mastitic milk. MDR was defined as acquired non-susceptibility to at least one agent in three or more antimicrobial categories. In the case of $S$. aureus, oxacillin or cefoxitin-resistant isolates were considered always as harboring MDR (Magiorakos et al., 2012).

\section{Statistical Analysis}

Microbiological data, recorded as $\mathrm{CFU} / \mathrm{ml}$, were transformed to logarithmic values before statistical analysis. The reported values of bacterial counts are the mean and 95\% CI values of the mean. Proportions to determine associations between bacterial species were compared using $\chi^{2}$ statistics. The frequencies of the susceptibility data for each antibiotic and microorganisms species were analyzed using a $\chi^{2}$ test followed by the Bonferroni post hoc pair-wise comparison. All analyses were conducted with a significance level of $p<0.05$. Collected data were analyzed using the statistical software StatGraphics Centurion XVI version 16.2.04 (StatPoint Technologies, Inc., Warrenton, VA, United States) and R 2.15.3 (R-project ${ }^{1}$ ).

\section{RESULTS}

\section{Microbiological Analysis of Milk Samples and Identification of Isolates by MALDI-TOF MS}

In this study, a total of 647 milk samples from women suffering infectious mastitis were analyzed using culture-based methods. Bacterial growth was observed in all analyzed samples with a mean value of $4.00 \log _{10} \mathrm{CFU} / \mathrm{ml}$ (95\% CI 3.94-4.06) and ranging from 1.70 to $6.21 \log _{10} \mathrm{CFU} / \mathrm{ml}$. A total of 2,323 isolates were identified by MALDI-TOF VITEK MS, 95.1\% at the species level and the remaining $4.9 \%$ at the genus level. The results are summarized in Table $\mathbf{1}$.

Staphylococcus was the most frequently isolated bacterial genus, since a total of 609 milk samples (94.1\%) contained at least one isolate of this genus. The total staphylococcal counts found in the milk samples presented a mean value of $3.70 \log _{10} \mathrm{CFU} / \mathrm{ml}$ (95\% CI 3.63-3.77). S. epidermidis was the most common species isolated from mastitis samples in this study (87.6\%), while S. aureus, S. hominis, and S. lugdunensis were isolated in 22.1, 5.3, and $3.3 \%$ of them, respectively. The mean bacterial counts for S. epidermidis and S. aureus were 3.56 (95\% CI 3.49-3.62) and $3.65 \log _{10} \mathrm{CFU} / \mathrm{ml}(95 \%$ CI 3.47-3.83), respectively. Rothia was present in $22.3 \%$ of the samples, being Rothia mucilaginosa the species most commonly isolated of this genus with a detection frequency of $17.3 \%$.

The total streptococcal counts in the samples had a mean value of $3.47 \log _{10} \mathrm{CFU} / \mathrm{ml}$ (95\% CI 3.40-3.55) and oscillated between 1.70 and $6.40 \log _{10} \mathrm{CFU} / \mathrm{ml}$. This genus was isolated from $68.6 \%$ of the samples and, therefore, constitutes the second most prevalent bacterial group among the samples of mastitis milk analyzed in this study. Streptococcal isolates belonging to the mitis group were detected in $49.2 \%$ of the samples, with S. mitis/oralis (40.8\%) and S. parasanguinis (14.4\%) as the most common species of this group. Salivarius group streptococci were

\footnotetext{
${ }^{1}$ http://www.r-project.org
} 
TABLE 1 | Microbiological analysis of milk samples from women suffering mastitis $(n=647)$.

\begin{tabular}{|c|c|c|c|c|}
\hline Microorganism & No. samples ${ }^{a}$ & Frequency (\%) & Mean $(95 \% \mathrm{Cl})\left(\log _{10} \mathrm{CFU} / \mathrm{mL}\right)$ & Range [min, $\max ]\left(\log _{10} \mathrm{CFU} / \mathrm{mL}\right)$ \\
\hline Staphylococcus epidermidis & 567 & 87.6 & $3.56(3.49-3.62)$ & $4.48[1.70,6.18]$ \\
\hline Staphylococcus aureus & 143 & 22.1 & $3.65(3.47-3.83)$ & $4.40[1.70,6.10]$ \\
\hline Staphylococcus hominis & 34 & 5.3 & $3.46(3.12-3.80)$ & $3.98[1.70,5.68]$ \\
\hline Staphylococcus lugdunensis & 21 & 3.3 & $3.18(2.75-3.58)$ & $3.30[1.70,5.00]$ \\
\hline Staphylococcus warneri & 11 & 1.7 & $2.79(2.61-2.97)$ & $0.88[2.30,3.18]$ \\
\hline Staphylococcus pasteuri & 10 & 1.6 & $3.28(2.79-3.78)$ & $2.12[2.18,4.30]$ \\
\hline Other staphylococcib & 16 & 2.5 & $3.02(2.64-3.42)$ & $2.60[1.70,4.30]$ \\
\hline Total staphylococci & 609 & 94.1 & $3.70(3.63-3.77)$ & $4.51[1.70,6.21]$ \\
\hline Rothia mucilaginosa & 112 & 17.3 & $2.62(2.49-2.75)$ & $2.78[1.70,4.48]$ \\
\hline Rothia spp. & 35 & 5.4 & $2.52(2.24-2.79)$ & $3.00[1.70,4.70]$ \\
\hline Total Rothia & 144 & 22.3 & $2.61(2.49-2.72)$ & $3.00[1.70,4.70]$ \\
\hline Streptococcus mitis/oralis & 264 & 40.8 & $3.27(3.17-3.37)$ & $4.30[1.70,6.00]$ \\
\hline Streptococcus parasanguinis & 93 & 14.4 & $3.29(3.14-3.44)$ & $3.30[1.70,5.00]$ \\
\hline Mitis group streptococci & 318 & 49.2 & 3.35 (3.26-3.44) & $4.30[1.70,6.00]$ \\
\hline Streptococcus salivarius & 238 & 36.8 & $3.05(2.95-3.14)$ & $3.54[1.70,5.24]$ \\
\hline Streptococcus vestibularis & 32 & 5.0 & $3.48(3.12-3.83)$ & $4.34[1.70,6.04]$ \\
\hline Salivarius group streptococci & 255 & 39.4 & $3.12(3.02-3.21)$ & $4.34[1.70,6.04]$ \\
\hline Other streptococcic & 68 & 10.5 & $3.36(3.14-3.59)$ & $4.70[1.70,6.40]$ \\
\hline Total streptococci & 444 & 68.6 & $3.47(3.40-3.55)$ & $4.70[1.70,6.40]$ \\
\hline Corynebacterium tuberculostearicum & 23 & 3.6 & $2.30(1.97-2.63)$ & $2.45[1.70,4.15]$ \\
\hline Corynebacterium kroppenstedtii & 13 & 2.0 & $2.17(1.94-2.41)$ & $1.00[1.70,2.70]$ \\
\hline Other corynebacteria $^{d}$ & 46 & 7.1 & $2.95(2.65-3.25)$ & $3.28[1.70,4.98]$ \\
\hline Total corynebacteria & 75 & 11.6 & $2.65(2.44-2.87)$ & $3.27[1.70,4.98]$ \\
\hline Enterococcie & 24 & 3.7 & $3.16(2.66-3.67)$ & $3.48[1.70,5.18]$ \\
\hline Total microorganisms & 647 & 100 & $4.00(3.94-4.06)$ & $4.52[1.70,6.21]$ \\
\hline
\end{tabular}

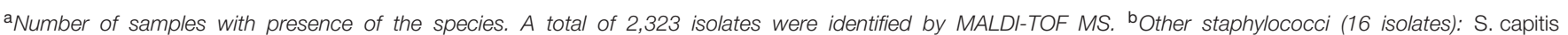

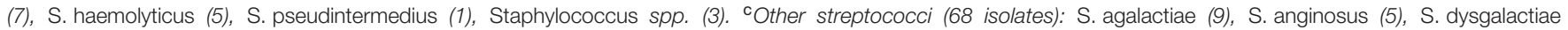

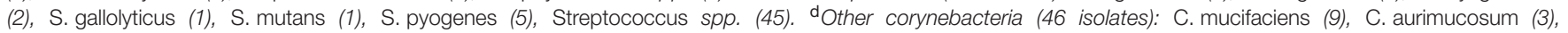

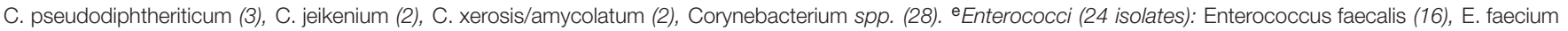
(4), Enterococcus spp. (4).

present in $39.4 \%$ of the samples, and the species S. salivarius and S. vestibularis had a detection frequency of 36.8 and $5 \%$, respectively.

Species belonging to the genus Corynebacterium were found in $11.6 \%$ of mastitis specimens, with a mean value of $2.65 \log _{10} \mathrm{CFU} / \mathrm{ml}$ (95\% CI 2.44-2.87). Corynebacterium tuberculostearicum (3.6\%) and Corynebacterium kroppenstedtii (2\%) were the corynebacterial species most frequently isolated in this study. Finally, enteroccocci were isolated in $3.7 \%$ of samples, being $E$. faecalis the most common species of this group.

\section{Antimicrobial Susceptibility Testing}

The antibiotic susceptibility profiles of 642 staphylococcal isolates are presented in Table 2. The MIC of the tested antimicrobial agents against $S$. aureus and $S$. epidermidis are compiled in Supplementary Tables S1, S2 and Figures S1, S2. Globally, the staphylococcal isolates displayed a remarkable resistance to benzylpenicillin (88.3\%) and erythromycin $(67.3 \%)$, although there were some differences depending on the species (Table 2). S. aureus isolates showed susceptibility (90-100\%) to most of the other antimicrobials tested, while S. epidermidis ones exhibited a noticeable percentage of resistance against oxacillin (46\%), cefoxitin (46\%), fusidic acid
(41.4\%), and mupirocin (28.7\%). No strains resistant to linezolid or tigecycline were found and the susceptibility to the rest of antibiotics tested was between 78.6 and 99.8\%. A significant difference between S. aureus and S. epidermidis isolates was detected for resistance to oxacillin, fusidic acid, mupirocin, and cefoxitin ( $\chi^{2}$ test, $p<0.001$ ), and benzylpenicillin, gentamycin, and clindamycin $\left(\chi^{2}\right.$ test, $\left.p<0.05\right)$. In addition to benzylpenicillin $(85.7 \%)$ and erythromycin (85.7\%), S. hominis isolates demonstrated remarkable resistance to fosfomycin $(81 \%)$, and moderate resistance to fusidic acid $(38.1 \%)$, oxacillin (33.3\%), cefoxitin (28.6\%), and clindamycin (23.8\%). Most of this species isolates were susceptible to the rest of the antimicrobials. All the isolates belonging to S. lugdunensis displayed high susceptibility against all the antibiotics tested (81-100\%), excepting benzylpenicillin (81\% resistant isolates).

The percentage of Staphylococcus isolates resistant to at least one antimicrobial and the number of resistances exhibited by these isolates was calculated for the most important species (Figure 1). The results indicated that a remarkable percentage of Staphylococcus isolates were resistant to at least one antibiotic: S. aureus (92.9\%), S. epidermidis (98.2\%), S. hominis (100\%), and S. lugdunensis (90.5\%). 
TABLE 2 | Antibiotic susceptibility profiles of S. aureus, S. epidermidis, and other Staphylococcus species isolated from milk samples from women suffering infectious mastitis.

\begin{tabular}{|c|c|c|c|c|c|c|c|}
\hline Antibiotic & & $\begin{array}{c}\text { All isolates } \\
(n=642)\end{array}$ & $\begin{array}{l}\text { S. aureus } \\
(n=140)\end{array}$ & $\begin{array}{l}\text { S. epidermidis } \\
(n=435)\end{array}$ & $\begin{array}{c}\text { S. hominis } \\
(n=21)\end{array}$ & $\begin{array}{l}\text { S. lugdunensis } \\
\quad(n=21)\end{array}$ & $\begin{array}{l}\text { Other* } \\
(n=25)\end{array}$ \\
\hline \multirow[t]{2}{*}{ Benzylpenicillin } & $\mathrm{R}$ & 88.3 & $81.4^{\dagger}$ & $92.9^{\dagger}$ & 85.7 & 81 & 54.2 \\
\hline & S & 11.7 & 18.6 & 7.1 & 14.3 & 19 & 45.8 \\
\hline \multirow[t]{2}{*}{ Oxacillin } & $\mathrm{R}$ & 32.8 & $2.9^{\ddagger}$ & $46^{\ddagger}$ & 33.3 & 0 & 0 \\
\hline & S & 67.2 & 97.1 & 54 & 66.7 & 100 & 100 \\
\hline \multirow[t]{2}{*}{ Gentamycin } & $\mathrm{R}$ & 3.9 & $\mathrm{O}^{\dagger}$ & $5.8^{\dagger}$ & 0 & 0 & 0 \\
\hline & S & 96.1 & 100 & 94.2 & 100 & 100 & 100 \\
\hline \multirow[t]{2}{*}{ Tobramycin } & $\mathrm{R}$ & 5.3 & 1.4 & 7.4 & 0 & 0 & 0 \\
\hline & S & 94.7 & 98.6 & 92.6 & 100 & 100 & 100 \\
\hline \multirow[t]{2}{*}{ Levofloxacin } & $\mathrm{R}$ & 4.4 & 1.4 & 6 & 0 & 0 & 0 \\
\hline & S & 95.6 & 98.6 & 94 & 100 & 100 & 100 \\
\hline \multirow[t]{2}{*}{ Erythromycin } & $\mathrm{R}$ & 67.3 & 65.7 & 71.5 & 85.7 & 19 & 28 \\
\hline & S & 32.7 & 34.3 & 28.5 & 14.3 & 81 & 72 \\
\hline \multirow[t]{2}{*}{ Clindamycin } & $\mathrm{R}$ & 17.9 & $10^{\dagger}$ & $21.4^{\dagger}$ & 23.8 & 14.3 & 0 \\
\hline & $S$ & 82.1 & 90 & 78.6 & 76.2 & 85.7 & 100 \\
\hline \multirow[t]{2}{*}{ Linezolid } & $\mathrm{R}$ & 0 & 0 & 0 & 0 & 0 & 0 \\
\hline & S & 100 & 100 & 100 & 100 & 100 & 100 \\
\hline \multirow[t]{2}{*}{ Daptomycin } & $\mathrm{R}$ & 0.2 & 0 & 0.2 & 0 & 0 & 0 \\
\hline & S & 99.8 & 100 & 99.8 & 100 & 100 & 100 \\
\hline \multirow[t]{2}{*}{ Teicoplanin } & $\mathrm{R}$ & 1.6 & 0 & 2.3 & 0 & 0 & 0 \\
\hline & S & 98.4 & 100 & 97.7 & 100 & 100 & 100 \\
\hline \multirow[t]{2}{*}{ Vancomycin } & $\mathrm{R}$ & 0.3 & 0 & 0.5 & 0 & 0 & 0 \\
\hline & S & 99.7 & 100 & 99.5 & 100 & 100 & 100 \\
\hline \multirow[t]{2}{*}{ Tigecycline } & $\mathrm{R}$ & 0.2 & 0 & 0 & 4.8 & 0 & 0 \\
\hline & S & 99.8 & 100 & 100 & 95.2 & 100 & 100 \\
\hline \multirow[t]{2}{*}{ Fosfomycin } & $\mathrm{R}$ & 12.1 & 2.9 & 6.9 & 81 & 14.3 & 96 \\
\hline & S & 87.9 & 97.1 & 93.1 & 19 & 85.7 & 4 \\
\hline \multirow[t]{2}{*}{ Fusidic acid } & $\mathrm{R}$ & 29.9 & $0^{\ddagger}$ & $41.4^{\ddagger}$ & 38.1 & 4.8 & 12 \\
\hline & S & 70.1 & 100 & 58.6 & 61.9 & 95.2 & 88 \\
\hline \multirow[t]{2}{*}{ Mupirocin } & $\mathrm{R}$ & 20.2 & $0^{\ddagger}$ & $28.7^{\ddagger}$ & 19 & 0 & 4 \\
\hline & S & 79.8 & 100 & 71.3 & 81 & 100 & 96 \\
\hline \multirow[t]{2}{*}{ Rifampicin } & $\mathrm{R}$ & 0.2 & 0 & 0.2 & 0 & 0 & 0 \\
\hline & S & 99.8 & 100 & 99.8 & 100 & 100 & 100 \\
\hline \multirow[t]{2}{*}{ TMP-SMZ } & $\mathrm{R}$ & 2.2 & 0 & 2.8 & 9.5 & 0 & 0 \\
\hline & S & 97.8 & 100 & 97.2 & 90.5 & 100 & 100 \\
\hline \multirow[t]{2}{*}{ Cefoxitin } & POS & 32.7 & $2.9^{\ddagger}$ & $46^{\ddagger}$ & 28.6 & 0 & 0 \\
\hline & NEG & 67.3 & 97.1 & 54 & 71.4 & 100 & 100 \\
\hline \multirow[t]{2}{*}{ ICR } & POS & 6.9 & 8.6 & 6.7 & 9.5 & 4.8 & 0 \\
\hline & NEG & 93.1 & 91.4 & 93.3 & 90.5 & 95.2 & 100 \\
\hline
\end{tabular}

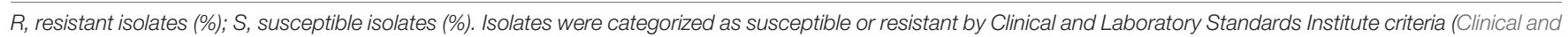

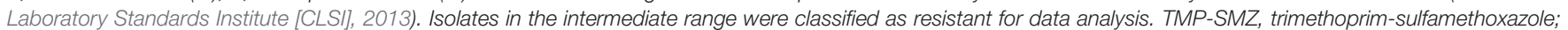

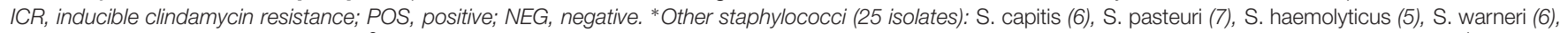

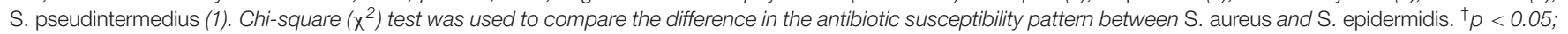
${ }^{\ddagger} p<0.001$.

S. epidermidis presented the highest number of antibiotic resistances (between 1 and 10), followed by $S$. hominis (16), S. aureus (1-5), and S. lugdunensis (1-4). The percentage of MDR isolates was noticeable in the case of $S$. hominis (81\%) and S. epidermidis (64.4\%). In relation to S. aureus and S. lugdunensis, 11.5 and $10.5 \%$ of MDR isolates were found, respectively.

Antimicrobial susceptibility tests were performed on a total of 524 streptococcal isolates and the results are summarized in Table 3. The MIC of the tested antimicrobial agents against S. mitis/oralis, S. parasanguinis, and S. salivarius are also presented in Supplementary Tables S3-S5 and Figures S3-S5. Overall, the streptococcal isolates showed high or moderate percentages of resistance to erythromycin (68.7\%), benzylpenicillin (63.7\%), ampicillin (51.5\%) and tetracycline (30.8\%), and susceptibility rates between 83.9 and $99.8 \%$ to the rest of the antimicrobials tested. S. mitis/oralis demonstrated a noteworthy resistance against erythromycin (69.6\%) and 


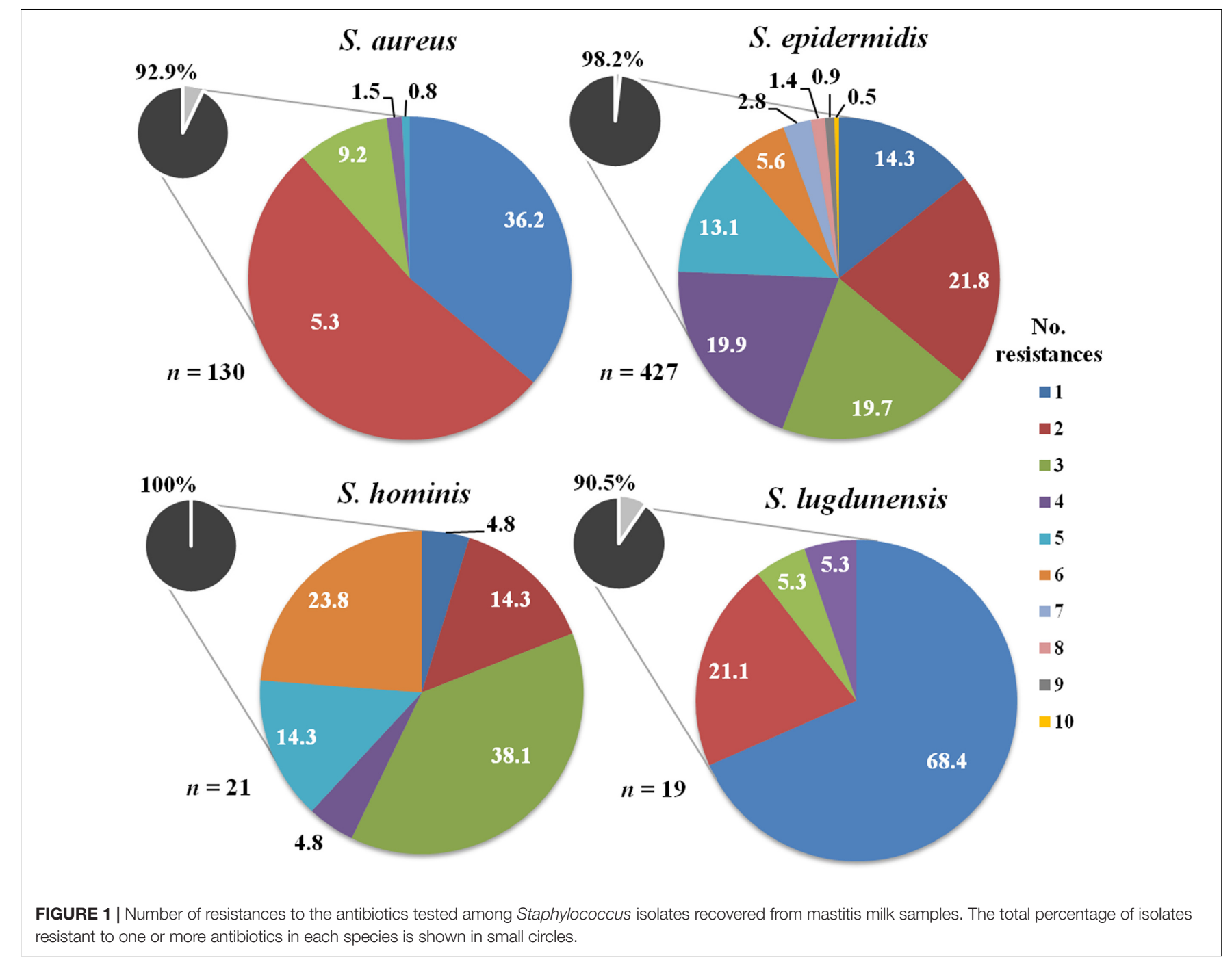

benzylpenicillin (56.7\%), followed by ampicillin (42.8\%) and tetracycline (30.7\%). Most of the isolates were sensitive to cefotaxime $(82.8 \%)$, ceftriaxone $(84.2 \%)$, clindamycin (89.3\%), levofloxacin (96.3\%), and linezolid (99.5\%). No vancomycin-resistant strains were identified. In comparison to $S$. mitis/oralis isolates, $S$. parasanguinis ones exhibited a higher percentage of resistance to erythromycin (80.8\%), benzylpenicillin (80.5\%), ampicillin (72\%), and tetracycline (51.2\%) and a slightly lower susceptibility to cefotaxime (79.3\%), ceftriaxone (81.7\%), clindamycin (82.9\%), and levofloxacin (85.4\%). No strains resistant to linezolid or vancomycin were found. S. salivarius also displayed remarkable resistance to benzylpenicillin (76.4\%), erythromycin (70.4\%), ampicillin (60.9\%), and a noticeable sensitivity (77.8-100\%) to the rest of antibiotics assayed. Regarding $S$. vestibularis, $41.4 \%$ of isolates were erythromycin-resistant; however, the susceptibility to the rest of antimicrobials tested was high (72.4-100\%). In fact, no strains resistant to cefotaxime, ceftriaxone, or linezolid appeared among the isolates of this species.
As shown in Figure 2, a high percentage of Streptococcus isolates from human mastitis samples were also resistant to at least one antibiotic: S. mitis/oralis (83.3\%), S. parasanguinis (97.6\%), S. salivarius (92.6\%), and S. vestibularis (72.4\%). S. salivarius presented the highest number of antibiotic resistances (between 1 and 9), followed by $S$. mitis/oralis (1-8), S. parasanguinis (1-7), and S. vestibularis (1-5). The percentage of MDR isolates was significant in the case of S. parasanguinis (51.2\%), S. salivarius (39.3\%) and S. mitis/oralis (34.6\%). Approximately, $19 \%$ of S. vestibularis isolates exhibited MDR.

Regarding the genus Enterococcus, E. faecalis isolates $(n=14)$ were not sensitive to those antibiotics to which they are intrinsically resistant (cefuroxime, cefuroximeaxetil, clindamycin, trimethoprim-sulfamethoxazole, and quinupristin-dalfopristin) (Table 4) and $85.7 \%$ of them were resistant to erythromycin. Approximately, $20 \%$ of the E. faecalis isolates displayed resistance to high levels of aminoglycosides (gentamycin, kanamycin, streptomycin) and around 7\% was resistant to quinolones (ciprofloxacin and levofloxacin) 
TABLE 3 | Antibiotic susceptibility profiles of Streptococcus species isolated from milk samples from women suffering infectious mastitis.

\begin{tabular}{|c|c|c|c|c|c|c|c|}
\hline Antibiotic & & $\begin{array}{c}\text { All isolates } \\
(n=524)\end{array}$ & $\begin{array}{l}\text { S. mitis/oralis } \\
(n=215)^{\ddagger}\end{array}$ & $\begin{array}{l}\text { S. parasanguinis } \\
\qquad(n=82)^{\ddagger}\end{array}$ & $\begin{array}{c}\text { S. salivarius } \\
(n=162)^{\ddagger}\end{array}$ & $\begin{array}{c}\text { S. vestibularis } \\
(n=29)\end{array}$ & $\begin{array}{c}\text { Other* } \\
(n=36)\end{array}$ \\
\hline \multirow[t]{2}{*}{ Benzylpenicillin } & $\mathrm{R}$ & 63.7 & $56.7^{\mathrm{a}}$ & $80.5^{\mathrm{b}}$ & $76.4^{\mathrm{b}}$ & 27.6 & 38.9 \\
\hline & S & 36.3 & 43.3 & 19.5 & 23.6 & 72.4 & 61.1 \\
\hline \multirow[t]{2}{*}{ Ampicillin } & $\mathrm{R}$ & 51.5 & $42.8^{a}$ & $71.9^{b}$ & $60.9^{b}$ & 24.1 & 37.1 \\
\hline & S & 48.5 & 57.2 & 28 & 39.1 & 75.9 & 62.9 \\
\hline \multirow[t]{2}{*}{ Cefotaxime } & $R$ & 14.8 & 17.2 & 20.7 & 9.9 & 0 & 24 \\
\hline & S & 85.2 & 82.8 & 79.3 & 90.1 & 100 & 76 \\
\hline \multirow[t]{2}{*}{ Ceftriaxone } & $R$ & 13 & 15.8 & 18.3 & 8 & 0 & 19.2 \\
\hline & S & 87 & 84.2 & 81.7 & 92 & 100 & 80.8 \\
\hline \multirow[t]{2}{*}{ Levofloxacin } & $\mathrm{R}$ & 12 & $3.7^{\mathrm{a}}$ & $14.6^{b}$ & $22.2^{\mathrm{c}}$ & 20.7 & 2.8 \\
\hline & S & 88 & 96.3 & 85.4 & 77.8 & 79.3 & 97.2 \\
\hline \multirow[t]{2}{*}{ Erythromycin } & $\mathrm{R}$ & 68.7 & 69.6 & 80.8 & 70.4 & 41.4 & 48.3 \\
\hline & S & 31.3 & 30.4 & 19.2 & 29.6 & 58.6 & 51.7 \\
\hline \multirow[t]{2}{*}{ Clindamycin } & $\mathrm{R}$ & 16.1 & 10.7 & 17.1 & 21.6 & 13.8 & 36.1 \\
\hline & S & 83.9 & 89.3 & 82.9 & 78.4 & 86.2 & 63.9 \\
\hline \multirow[t]{2}{*}{ Linezolid } & $\mathrm{R}$ & 0.2 & 0.5 & 0 & 0 & 0 & 0 \\
\hline & S & 99.8 & 99.5 & 100 & 100 & 100 & 100 \\
\hline \multirow[t]{2}{*}{ Vancomycin } & $\mathrm{R}$ & 1 & 0 & 0 & 2.5 & 3.4 & 0 \\
\hline & $S$ & 99 & 100 & 100 & 97.5 & 96.6 & 100 \\
\hline \multirow[t]{2}{*}{ Tetracycline } & $\mathrm{R}$ & 30.8 & $30.7^{a}$ & $51.2^{\mathrm{b}}$ & $21^{a}$ & 17.2 & 38.5 \\
\hline & $S$ & 69.2 & 69.3 & 48.8 & 79 & 82.8 & 61.5 \\
\hline
\end{tabular}

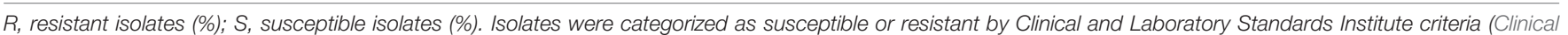

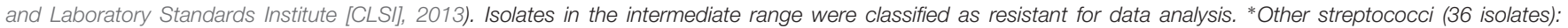

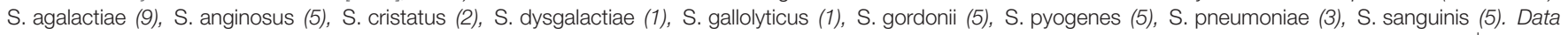

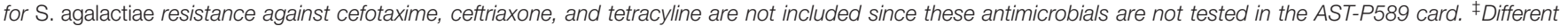

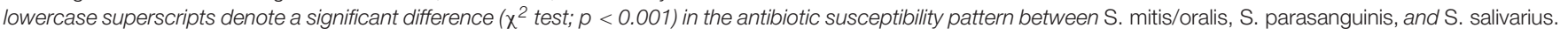

and glycopeptides (vancomycin and teicoplanin). Globally, 93\% of E. faecalis isolates were resistant to, at least, one antibiotic (excluding intrinsic resistance) and $21.4 \%$ were MDR (Figure 3).

\section{DISCUSSION}

Mastitis is a common reason for antibiotic treatment in lactating women. In fact, studies carried out in industrialized countries have reported high rates of antibiotic prescriptions for mastitis (Foxman et al., 2002; Scott et al., 2008), despite the fact that this empiric practice may be a relevant risk factor for acute or subacute episodes to become chronic or recurrent due to the selection of antibiotic resistant strains (Mediano et al., 2014; Fernández et al., 2016). In the absence of microbiological analysis and antimicrobial susceptibility testing, the antibiotic treatment usually depends on physician choice rather than on scientific evidence, leading to the increase of antimicrobial resistance among mastitis-causing agents (Barlow, 2011; Jahanfar et al., 2013).

It is widely accepted that antibiotics are responsible for dysbiosis processes in human microbiota leading to antibioticassociated diarrhea and gastroenteritis, urogenital and oral infections. Host-microbiota interactions are dynamic and, therefore, changes in the microbiota as a consequence of antibiotic treatment can result in the dysregulation of host immune homeostasis and an increased susceptibility to infectious, allergic and inflammatory diseases (Joffe and Simpson, 2009; Willing et al., 2011). In the last few years, it is becoming evident that antibiotic therapy during pregnancy, intrapartum or lactation affects the maternal (intestinal, vaginal, mammary) microbiota (Soto et al., 2014; Gonçalves et al., 2016), which alters normal mother-to-infant transmission of microorganisms, affecting the development of the infant intestinal microbiota and having negative consequences to infant health (Murk et al., 2011; Stensballe et al., 2013; Gibson et al., 2015; Rutten et al., 2015; Mazzola et al., 2016).

Nowadays, the most commonly prescribed antibiotics for mastitis are penicillins, cephalosporins, erythromycin, and clindamycin (Foxman et al., 2002; Eglash et al., 2006; Scott et al., 2008; Barlow, 2011; Amir and Academy of Breastfeeding Medicine Protocol Committee, 2014; Witt et al., 2014); however, there is limited consensus on which patients should receive antibiotics, which one is the most appropriate, when to initiate therapy, its duration or the dosage and posology (Jahanfar et al., 2013; Amir and Academy of Breastfeeding Medicine Protocol Committee, 2014). Moreover, the impact of antibiotic therapy on the host microbiota and on the development of antimicrobial resistance among potential human mastitis pathogens has been scarcely evaluated to date.

Staphylococci are the most common pathogens causing human mastitis (Lawrence and Lawrence, 2011); S. aureus is the main etiological agent of acute mastitis and breast abscesses (Reddy et al., 2007; Delgado et al., 2011; 


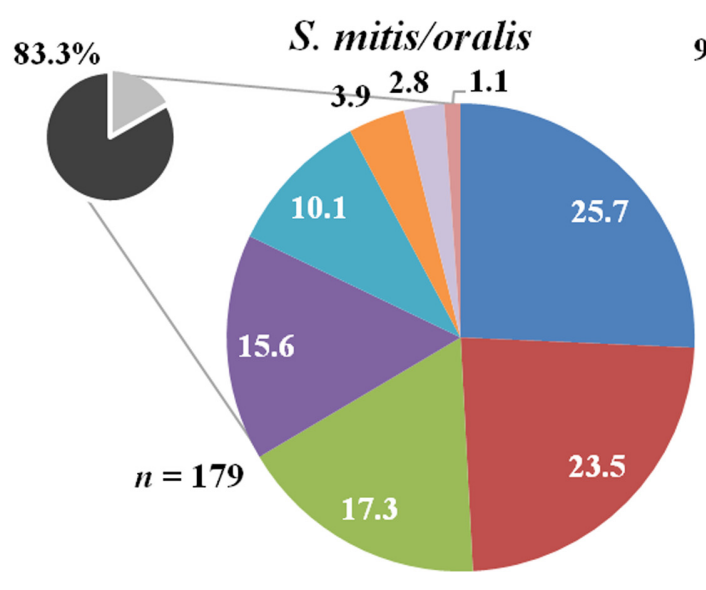

\section{6\% S.parasanguinis}
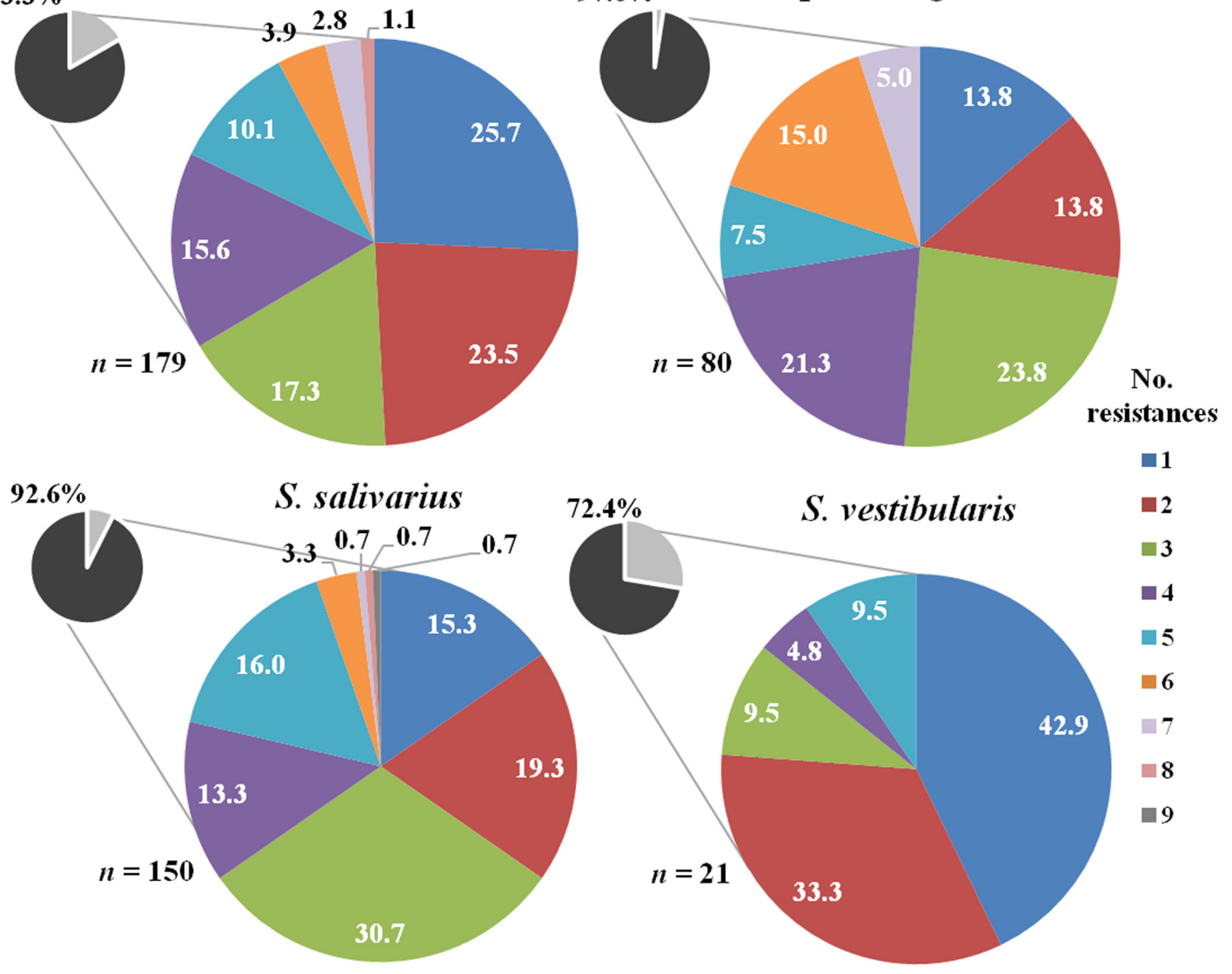

S. vestibularis

$\square 2$

$\square 3$

$\square 4$

$\square$

6

$\square 7$

$\mathbf{8}$

$\square 9$

FIGURE 2 | Number of resistances to the antibiotics tested among Streptococcus isolates recovered from mastitis milk samples. The total percentage of isolates resistant to one or more antibiotics in each species is shown in small circles.

Branch-Elliman et al., 2013), while coagulase-negative staphylococci are usually related to subacute or subclinical infection. Among the latter, S. epidermidis is emerging as the leading cause of mastitis worldwide (Contreras and Rodríguez, 2011). In this sense, the results of the present study confirm the important role that $S$. epidermidis plays as an etiologic agent in human mastitis, in agreement with previous studies (Delgado et al., 2008, 2009; Mediano et al., 2017).

There are limited studies reporting the antimicrobial susceptibility of staphylococci isolates recovered from milk of women suffering mastitis. In the present study, antimicrobial resistance among the $S$. aureus isolates was less common than among the $S$. epidermidis ones, although the resistance to benzylpenicillin and erythromycin among all staphylococcal isolates was remarkable. First generation penicillins, such as amoxicillin, and the macrolide erythromycin have been recommended for years (WHO, 2000), but nowadays their use is discouraged due to the increasing resistance to these antibiotics among S. aureus isolates (Scott et al., 2008). Clinicians should also be aware of the rise of methicillin-resistant Staphylococcus aureus (MRSA) isolated in cases of mastitis and breast abscesses (Reddy et al., 2007; Branch-Elliman et al., 2013). However, in this study, S. aureus isolates showed high susceptibility to oxacillin, in accordance with a previous work (Delgado et al., 2011). Furthermore, among the isolates of $S$. aureus that exhibited some resistance, about $12 \%$ were $\mathrm{MDR}$, in contrast to S. epidermidis (64\%). The evolution of antibiotic resistance among S. aureus involved in mastitis should be followed up since this species has a remarkable ability to acquire multi-antibiotic resistance (Hiramatsu et al., 2014).

Penicillin resistance is the most common antimicrobial resistance in pathogenic S. epidermidis (Bansal et al., 2015; Taponen et al., 2016). In this study, the highest antibiotic resistance of this species was observed against benzylpenicillin, which is conferred by the production of $\beta$-lactamases, but the resistance to oxacillin, which is resistant to these enzymes, must be highlighted. In this sense, rates of methicillin resistance in $S$. epidermidis have increased steadily during the last decades (Becker et al., 2014), which is of special concern because mec genes may be transferred between staphylococcal species and constitute a risk for public health (Wendlandt et al., 2013; Taponen et al., 2016). Delgado et al. (2009) characterized the antimicrobial susceptibility profiles of $40 \mathrm{~S}$. epidermidis strains from human mastitis and showed a high percentage of resistance 
TABLE 4 | Antibiotic susceptibility profiles of Enterococcus faecalis isolated from human mastitis.

\begin{tabular}{|c|c|c|}
\hline Antibiotic & & E. faecalis $(n=14)$ \\
\hline \multirow[t]{2}{*}{ Benzylpenicillin } & $\mathrm{R}$ & 7.1 \\
\hline & S & 92.9 \\
\hline \multirow[t]{2}{*}{ Ampicillin } & $\mathrm{R}$ & 7.7 \\
\hline & S & 92.3 \\
\hline \multirow[t]{2}{*}{ Cefuroxime } & $\mathrm{R}$ & 100 \\
\hline & S & 0 \\
\hline \multirow[t]{2}{*}{ Cefuroxime-axetil } & $\mathrm{R}$ & 100 \\
\hline & S & 0 \\
\hline \multirow[t]{2}{*}{ Imipenem } & $\mathrm{R}$ & 0 \\
\hline & S & 100 \\
\hline \multirow[t]{2}{*}{ Gentamycin HL } & $\mathrm{R}$ & 16.7 \\
\hline & S & 83.3 \\
\hline \multirow[t]{2}{*}{ Kanamycin HL } & $\mathrm{R}$ & 23.1 \\
\hline & S & 76.9 \\
\hline \multirow[t]{2}{*}{ Streptomycin HL } & $\mathrm{R}$ & 25 \\
\hline & $S$ & 75 \\
\hline \multirow[t]{2}{*}{ Ciprofloxacin } & $\mathrm{R}$ & 7.7 \\
\hline & S & 92.3 \\
\hline \multirow[t]{2}{*}{ Levofloxacin } & $\mathrm{R}$ & 7.1 \\
\hline & S & 92.9 \\
\hline \multirow[t]{2}{*}{ Erythromycin } & $\mathrm{R}$ & 85.7 \\
\hline & S & 14.3 \\
\hline \multirow[t]{2}{*}{ Clindamycin } & $\mathrm{R}$ & 100 \\
\hline & $S$ & 0 \\
\hline \multirow[t]{2}{*}{ QD } & $\mathrm{R}$ & 100 \\
\hline & S & 0 \\
\hline \multirow[t]{2}{*}{ Linezolid } & $\mathrm{R}$ & 0 \\
\hline & S & 100 \\
\hline \multirow[t]{2}{*}{ Teicoplanin } & $\mathrm{R}$ & 7.1 \\
\hline & $S$ & 92.9 \\
\hline \multirow[t]{2}{*}{ Vancomycin } & $\mathrm{R}$ & 7.7 \\
\hline & S & 92.3 \\
\hline \multirow[t]{2}{*}{ Tigecycline } & $\mathrm{R}$ & 0 \\
\hline & S & 100 \\
\hline \multirow[t]{2}{*}{ Nitrofurantoin } & $\mathrm{R}$ & 7.7 \\
\hline & S & 92.3 \\
\hline \multirow[t]{2}{*}{ Chloramphenicol } & $\mathrm{R}$ & 0 \\
\hline & $S$ & 100 \\
\hline \multirow[t]{2}{*}{ TMP-SMZ } & $\mathrm{R}$ & 100 \\
\hline & S & 0 \\
\hline
\end{tabular}

$\overline{R \text {, resistant isolates (\%); S, susceptible isolates (\%); HL, high level; QD, quinupristin- }}$ dalfopristin; TMP-SMZ, trimethoprim-sulfamethoxazole.

to oxacillin, erythromycin, clindamycin and mupirocin, in agreement with the results of the present study. Similar resistance frequency for oxacillin and clindamycin have been described in the case of coagulase-negative staphylococci isolated from milk of lactating women with chronic breast pain receiving oral antibiotic therapy (Witt et al., 2014). Among the findings of this work, it has to be highlighted the high number of antibiotic resistances displayed by some isolates of S. epidermidis (up to 10) and $S$. hominis (up to six) and the high percentage of MDR isolates, about $64 \%$ and $81 \%$, respectively. Frequent resistance to several antimicrobials has been reported for these species in previous studies (Sahal and Bilkay, 2014; Deplano et al., 2016; Szczuka et al., 2016).

Streptococci constitute the second bacterial group involved in human infectious mastitis as suggested by the results of this study. Most streptococci isolated from mastitis samples belonged to the VGS, mainly to mitis and salivarius groups, as previously reported (Delgado et al., 2008; Arroyo et al., 2010; Mediano et al., 2017). These microorganisms are normally related to subacute and subclinical processes (WHO, 2000; Contreras and Rodríguez, 2011). Similarly to coagulasenegative staphylococci, VGS have been considered commensal microorganisms and, independent of their concentration and strain characteristics, they have been regarded as contaminants; as a consequence, their clinical relevance has often been questioned.

Accurate identification of VGS isolates at the species level is becoming an important issue to provide a better understanding of their potential pathogenicity and the increasing antibiotic resistance observed among them (Bruckner and Gigliotti, 2006; Doern and Burnham, 2010; Chun et al., 2015). The fact that many clinical and epidemiological data concerning these organisms usually refer to the entire VGS group rather than to a single species (Gordon et al., 2002) is an obstacle for knowing the role of each species of the group. Recently, the application of the MALDI-TOF MS has emerged as a useful, rapid and costeffective method for the identification of these clinically relevant microorganisms (Kärpänoja et al., 2014; Angeletti et al., 2015; Martín et al., 2016).

Knowledge of antibiotic resistance in VGS is more limited than for staphylococci. Indeed, the antimicrobial susceptibility of streptococci isolates recovered from human mastitis has not been described to date. The increasing resistance of VGS against penicillin, erythromycin and tetracycline has become a concerning issue in the clinical practice (Gordon et al., 2002; Bruckner and Gigliotti, 2006; Ergin et al., 2006; Doern and Burnham, 2010; Chun et al., 2015), which is according with the high levels of resistance to these antimicrobials found among VGS isolates from human mastitis cases. Erythromycin-resistant VGS isolates usually are also resistant to tetracycline (Ergin et al., 2006), since the resistance genes to these antibiotics are often found on the same mobile genetic element (Cerdá Zolezzi et al., 2004; Doern and Burnham, 2010).

There were significant differences regarding the antibiotic susceptibility profiles among the different VGS species isolated in this study. S. parasanguinis exhibited a significantly higher resistance to benzylpenicillin, ampicillin, and tetracycline than $S$. salivarius and $S$. mitis/oralis, with antibiotic resistance percentages consistent with those reported by Chun et al. (2015). In relation to $S$. salivarius, the resistance to penicillins and levofloxacin was also higher than that demonstrated by $S$. mitis/oralis. These results are in disagreement with previous studies that have addressed higher antibiotic resistance in S. mitis/oralis compared with other streptococci (Han et al., 2006). Results concerning MDR revealed than $51 \%$ of the $S$. parasanguinis isolates were MDR compared to $39 \%$ of $S$. salivarius and $35 \%$ of S. mitis/oralis. This is in 


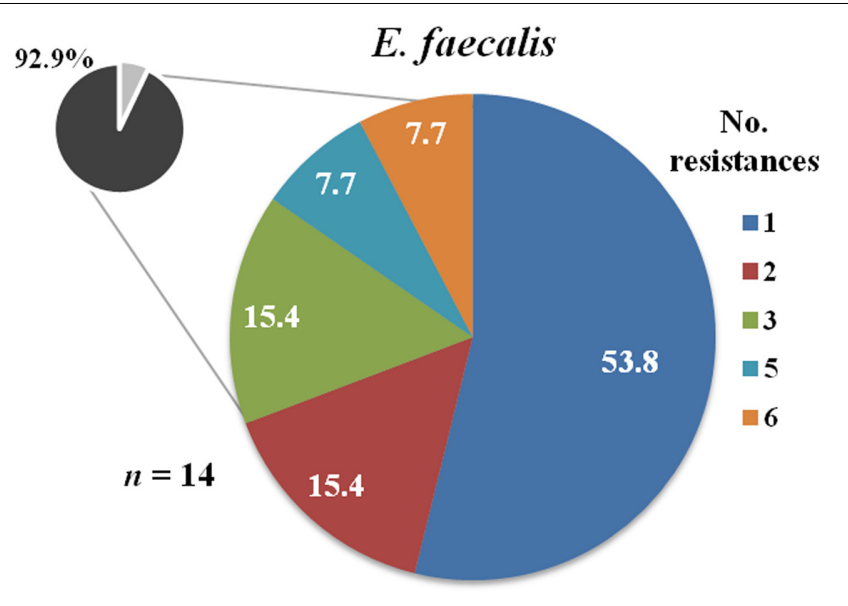

FIGURE 3 | Number of resistances to the antibiotics tested among Enterococcus faecalis isolates recovered from mastitis milk samples. The total percentage of isolates resistant to one or more antibiotics is shown in the small circle (intrinsic resistances to cefuroxime, cefuroxime-axetil, clindamycin, trimethoprim-sulfamethoxazole, and quinupristin-dalfopristin are excluded).

contrast with previous studies describing that S. mitis/oralis was more resistant to antibiotics than other VGS species (Doern and Burnham, 2010). S. parasanguinis and S. salivarius may play a relevant role in antibiotic resistance transmission among VGS involved in human mastitis, a fact that deserves to be addressed in the future.

The resistance patterns reported for staphylococci and streptococci in this study are concordant with commonly prescribed antibiotics, which supports that selective pressure from the use of antibiotics is a main factor in the development of antibiotic resistance (Fair and Tor, 2014), as well as in the molecular changes that enhance the virulence and biofilmforming ability of different microorganisms (Dancer, 2004; Sahal and Bilkay, 2014). Therefore, the development of antimicrobial resistance among staphylococci and VGS isolated from human mastitis has serious implications for the treatment of this condition. Benzylpenicillin and erythromycin resistance is so high among these bacterial groups that they and closely related molecules should not be considered as a treatment of choice. On the other hand, the prevalence of oxacillin resistance among S. epidermidis isolates must also be contemplated as an issue of clinical relevance in relation to treatment options. Regarding streptococci, the differences in antimicrobial resistance pattern among species must be taken into consideration to decide the most appropriate antimicrobial therapy.

In practice, in vitro sensitivity to a given antibiotic does not mean necessarily that such antibiotic will be efficient for the treatment of mastitis. It must be highlighted that, beside antimicrobial resistance, other factors may lead to failure of antibiotic therapy and persistence of infection and, among them, the biofilm formation ability of the strain involved is of utmost importance (Høiby et al., 2010; Büttner et al., 2015). $S$. epidermidis is commonly regarded as the most frequent causative agent of infections of indwelling medical devices (such as peripheral or central venous catheters), followed by $S$. aureus. Once the bacteria have entered the body, they use various virulence factors to facilitate interactions with host tissues and subvert the host's immune system. Interestingly, lactating mammary glands contain an extraordinary complex net of ducts during lactation that provides an excellent physical support for biofilm formation and maturation. Biofilms are multicellular, surface-attached agglomerations of microorganisms and their regulation involves quorum-sensing systems and it is not yet completely understood. Previous epidemiological and genetic studies suggest that $S$. epidermidis isolates in the clinical environment differ from those obtained outside of medical facilities in terms of biofilm formation, antibiotic resistance, and the presence of mobile DNA elements (Otto, 2009). In this context, the comparison of several properties of 200 $S$. epidermidis strains isolated from women with mastitis with those displayed by 105 ones isolated from milk of healthy women revealed that the number of strains that contained biofilm-related genes and that showed resistance to oxacillin, erythromycin, clindamycin, and mupirocin was significantly higher among the strains isolated from mastitic milk (Delgado et al., 2009). The authors suggested that resistance to diverse antibiotics and a greater ability to form biofilms found among the clinical strains may explain the chronic and recurrent nature of this infectious condition.

The genera Corynebacterium and Rothia are increasingly recognized as emerging opportunistic pathogens (Bernard, 2012; Ramanan et al., 2014), but their relevance in human mastitis may be greatly underestimated. Nevertheless, corynebacteria are common mastitis agents in ruminants (Contreras and Rodríguez, 2011) and they are the main cause of human granulomatous mastitis (Taylor et al., 2003; Mathelin et al., 2005). Rothia spp. have emerged as opportunistic pathogens associated with serious infections including sepsis, endocarditis, and meningitis in immune-compromised patients (Ramanan et al., 2014; Abidi et al., 2016). R. mucilaginosa, the most common species of this genus isolated in this study, has been previously involved in prosthetic device infections, which could be related, as in the case of coagulase-negative staphylococci, to the organism's ability to produce a biofilm (Bruminhent et al., 2013). In this study, Corynebacterium and Rothia were found in 11 and $22 \%$ of samples, respectively, which suggests an important role in human mastitis pathogenesis, according to a previous study (Mediano et al., 2017). In relation to antimicrobial susceptibility, the VITEK 2 technology used for AST in this study is not available for these bacterial genera yet, and therefore the analyses of their antimicrobial resistance profiles will be part of a future study. It is of utmost importance since many medically relevant species have shown broad-spectrum antibiotic resistance which has important therapeutic implications (Olender, 2013; Dobinson et al., 2015).

Enteroccoccus are not frequent etiological agents in human mastitis but they can be relevant in specific cases. Isolates of this genus were present in some samples of this study $(3.7 \%)$, most of them belonging to E. faecalis. Treatment of enterococcal mastitis may be particularly challenging due to the wide variety of antibiotic mechanisms displayed by the genus (Sood et al., 2008; 
Kristich et al., 2014). The antimicrobial susceptibility patterns of enterococci isolated from human mastitis have not been reported to date. In this study, the high level resistance to aminoglycosides exhibited by about $20 \%$ of isolates, as well as the remarkable resistance to erythromycin (86\%), is in concordance with previous studies (Kristich et al., 2014; Chakraborty et al., 2015; Osuka et al., 2016). On the contrary, all isolates showed high susceptibility to ampicillin as reported for enterococci isolated from mastitic bovine milk (Nam et al., 2010). It must also be addressed that about $7 \%$ of the E. faecalis isolates obtained in this study showed resistance to quinolones and vancomycin. Even though E. faecalis resistance is low against vancomycin, the emergence of vancomycin-resistant enterococci is a cause of concern, as it can be transferred to S. aureus (Sood et al., 2008).

\section{CONCLUSION}

The findings of this work are relevant to identify trends in antimicrobial resistance among mastitis pathogens, including the emergence of MDR strains, and to implement a rational treatment for this disease based on microbiological analysis and antibiotic susceptibility profiling. Further work is in progress to analyze the value of implementing AST on the clinical outcomes of women treated for mastitis, as well as to investigate the genetic mechanisms underlying the antimicrobial resistance.

\section{REFERENCES}

Abidi, M. Z., Ledeboer, N., Banerjee, A., and Hari, P. (2016). Morbidity and mortality attributable to Rothia bacteremia in neutropenic and nonneutropenic patients. Diagn. Microbiol. Infect. Dis. 85, 116-120. doi: 10.1016/j.diagmicrobio. 2016.01.005

Amir, L. H., and Academy of Breastfeeding Medicine Protocol Committee (2014). ABM clinical protocol \#4: Mastitis, revised March 2014. Breastfeed. Med 9, 239-243. doi: 10.1089/bfm.2014.9984

Angeletti, S., Dicuonzo, G., Avola, A., Crea, F., Dedej, E., Vailati, F., et al. (2015). Viridans Group Streptococci clinical isolates: MALDI-TOF mass spectrometry versus gene sequence-based identification. PLOS ONE 10:e0120502. doi: 10. 1371/journal.pone.0120502

Arroyo, R., Martín, V., Maldonado, A., Jiménez, E., Fernández, L., and Rodríguez, J. M. (2010). Treatment of infectious mastitis during lactation: antibiotics versus oral administration of Lactobacilli isolated from breast milk. Clin. Infect. Dis. 50, 1551-1558. doi: 10.1086/652763

Bansal, B. K., Gupta, D. K., Shafi, T. A., and Sharma, S. (2015). Comparative antibiogram of coagulase-negative Staphylococci (CNS) associated with subclinical and clinical mastitis in dairy cows. Vet. World 8, 421-426. doi: 10.14202/vetworld.2015.421-426

Barlow, J. (2011). Mastitis therapy and antimicrobial susceptibility: a multispecies review with a focus on antibiotic treatment of mastitis in dairy cattle. J. Mammary Gland Biol. Neoplasia 16, 383-407. doi: 10.1007/s10911-0119235-Z

Becker, K., Heilmann, C., and Peters, G. (2014). Coagulase-negative staphylococci. Clin. Microbiol. Rev. 27, 870-926. doi: 10.1128/CMR.00109-13

Bernard, K. (2012). The genus Corynebacterium and other medically relevant coryneform-like bacteria. J. Clin. Microbiol. 50, 3152-3158. doi: 10.1128/JCM. 00796- 12

Bizzini, A., Jaton, K., Romo, D., Bille, J., Prod'hom, G., and Greub, G. (2011). Matrix-assisted laser desorption ionization-time of flight mass spectrometry as an alternative to $16 \mathrm{~S}$ rRNA gene sequencing for identification of difficultto-identify bacterial strains. J. Clin. Microbiol. 49, 693-696. doi: 10.1128/JCM. 01463-10

\section{AUTHOR CONTRIBUTIONS}

MM: database construction, data analysis and interpretation, and wrote the paper. RA: bacterial identification, antimicrobial susceptibility testing. IE-M: data analysis. LF, JR: designed the research and reviewed the paper.

\section{FUNDING}

This study was funded by the Ministerio de Economía y Competitividad (Spain) (AGL2013-41980-P and AGL201675476-R projects).

\section{ACKNOWLEDGMENT}

We thank Manuela Pérez for her valuable help with the database construction.

\section{SUPPLEMENTARY MATERIAL}

The Supplementary Material for this article can be found online at: http://journal.frontiersin.org/article/10.3389/fmicb. 2017.01258/full\#supplementary-material

Branch-Elliman, W., Lee, G. M., Golen, T. H., Gold, H. S., Baldini, L. M., and Wright, S. B. (2013). Health and economic burden of post-partum Staphylococcus aureus breast abscess. PLoS ONE 8:e73155. doi: 10.1371/journal. pone.0073155

Bruckner, L., and Gigliotti, F. (2006). Viridans group streptococcal infections among children with cancer and the importance of emerging antibiotic resistance. Semin. Pediatr. Infect. Dis. 17, 153-160. doi: 10.1053/j.spid.2006. 06.008

Bruminhent, J., Tokarczyk, M. J., Jungkind, D., and DeSimone, J. A. (2013). Rothia mucilaginosa prosthetic device infections: a case of prosthetic valve endocarditis. J. Clin. Microbiol. 51, 1629-1632. doi: 10.1128/JCM.031 73-12

Büttner, H., Mack, D., and Rohde, H. (2015). Structural basis of Staphylococcus epidermidis biofilm formation: mechanisms and molecular interactions. Front. Cell. Infect. Microbiol. 5:14. doi: 10.3389/fcimb.2015.00014

Cerdá Zolezzi, P., Laplana, L. M., Calvo, C. R., Cepero, P. G., Erazo, M. C., and Gómez-Lus, R. (2004). Molecular basis of resistance to macrolides and other antibiotics in commensal viridans group streptococci and Gemella spp. and transfer of resistance genes to Streptococcus pneumoniae. Antimicrob. Agents Chemother. 48, 3462-3467. doi: 10.1128/AAC.48.9.3462-3467. 2004

Chakraborty, A., Pal, N. K., Sarkar, S., and Gupta, M. S. (2015). Antibiotic resistance pattern of Enterococci isolates from nosocomial infections in a tertiary care hospital in Eastern India. J. Nat. Sci. Biol. Med. 6, 394-397. doi: 10.4103/09769668.160018

Chun, S., Huh, H. J., and Lee, N. Y. (2015). Species-specific difference in antimicrobial susceptibility among viridans group streptococci. Ann. Lab. Med. 35, 205-211. doi: 10.3343/alm.2015.35.2.205

Clinical and Laboratory Standards Institute [CLSI] (2013). Performance Standards for Antimicrobial Susceptibility Testing; Twenty-Third Informational Supplement. CLSI document M100-S23. Wayne, PA: Clinical Laboratory Standards Institute.

Contreras, G. A., and Rodríguez, J. M. (2011). Mastitis: comparative etiology and epidemiology. J. Mammary Gland Biol. Neoplasia 16, 339-356. doi: 10.1007/ s10911-011-9234-0 
Dancer, S. J. (2004). How antibiotics can make us sick: the less obvious adverse effects of antimicrobial chemotherapy. Lancet Infect. Dis. 4, 611-619. doi: 10.1016/S1473-3099(04)01145-4

Delgado, S., Arroyo, R., Jiménez, E., Marín, M. L., del Campo, R., Fernández, L., et al. (2009). Staphylococcus epidermidis strains isolated from breast milk of women suffering infectious mastitis: potential virulence traits and resistance to antibiotics. BMC Microbiol. 9:82. doi: 10.1186/1471-2180-9-82

Delgado, S., Arroyo, R., Martín, R., and Rodríguez, J. M. (2008). PCR-DGGE assessment of the bacterial diversity of breast milk in women with lactational infectious mastitis. BMC Infect. Dis. 8:51. doi: 10.1186/1471-2334-8-51

Delgado, S., García, P., Fernández, L., Jiménez, E., Rodríguez-Baños, M., del Campo, R., et al. (2011). Characterization of Staphylococcus aureus strains involved in human and bovine mastitis. FEMS Immunol. Med. Microbiol. 62, 225-235. doi: 10.1111/j.1574-695X.2011.00806.x

Delgado, S., García-Garrote, F., Padilla, B., Rodríguez Gómez, J. M., and Romero, B. (2015). "Microbiological diagnosis of bacterial infection associated with delivery and postpartum [in Spanish]," in Procedures in Clinical Microbiology (number 54), eds E. Cercenado and R. Cantón. Spanish Society of Infectious Diseases and Clinical Microbiology. Available at: http://www.seimc.org/contenidos/documentoscientificos/procedimientosmicro biologia/seimc-procedimientomicrobiologia54.pdf [accessed June 27, 2017].

Deplano, A., Vandendriessche, S., Nonhoff, C., Dodémont, M., Roisin, S., and Denis, O. (2016). National surveillance of Staphylococcus epidermidis recovered from bloodstream infections in Belgian hospitals. J. Antimicrob. Chemother 71, 1815-1819. doi: 10.1093/jac/dkw086

Dobinson, H. C., Anderson, T. P., Chambers, S. T., Doogue, M. P., Seaward, L., and Werno, A. M. (2015). Antimicrobial treatment options for granulomatous mastitis caused by Corynebacterium species. J. Clin. Microbiol 53, 2895-2899. doi: 10.1128/JCM.00760-15

Doern, C. D., and Burnham, C.-A. D. (2010). It's not easy being green: the viridans group streptococci, with a focus on pediatric clinical manifestations. J. Clin. Microbiol. 48, 3829-3835. doi: 10.1128/JCM.01563-10

Eglash, A., Plane, M. B., and Mundt, M. (2006). History, physical and laboratory findings, and clinical outcomes of lactating women treated with antibiotics for chronic breast and/or nipple pain. J. Hum. Lact. 22, 429-433. doi: 10.1177/ 0890334406293431

Ergin, A., Ercis, S., and Hasçelik, G. (2006). Macrolide resistance mechanisms and in vitro susceptibility patterns of viridans group streptococci isolated from blood cultures. J. Antimicrob. Chemother. 57, 139-141. doi: 10.1093/jac/dki404

Fair, R. J., and Tor, Y. (2014). Antibiotics and bacterial resistance in the 21st century. Perspect. Med. Chem. 6, 25-64. doi: 10.4137/PMC.S14459

Fernández, L., Langa, S., Martín, V., Maldonado, A., Jiménez, E., Martín, R., et al. (2013). The human milk microbiota: Origin and potential roles in health and disease. Pharmacol. Res. 69, 1-10. doi: 10.1016/j.phrs.2012. 09.001

Fernández, L., Mediano, P., García, R., Rodríguez, J. M., and Marín, M. (2016). Risk factors predicting infectious lactational mastitis: decision tree approach versus logistic regression analysis. Matern. Child Health J. 20, 1895-1903. doi: 10.1007/s10995-016-2000-6

Fernández, L., and Rodríguez, J. M. (eds) (2014). Mastitis, el lado oscuro de la lactancia. Microbiota mamaria: de la fisiología a las mastitis. ISBN: 978-84-6171164-2. Probisearch, Madrid.

Foxman, B., D’Arcy, H., Gillespie, B., Bobo, J. K., and Schwartz, K. (2002). Lactation mastitis: occurrence and medical management among 946 breastfeeding women in the United States. Am. J. Epidemiol. 155, 103-114. doi: 10.1093/aje/ 155.2.103

Gibson, M. K., Crofts, T. S., and Dantas, G. (2015). Antibiotics and the developing infant gut microbiota and resistome. Curr. Opin. Microbiol. 27, 51-56. doi: 10.1016/j.mib.2015.07.007

Gonçalves, B., Ferreira, C., Alves, C. T., Henriques, M., Azeredo, J., and Silva, S. (2016). Vulvovaginal candidiasis: Epidemiology, microbiology and risk factors. Crit. Rev. Microbiol. 42, 905-927. doi: 10.3109/1040841X.2015. 1091805

Gordon, K. A., Beach, M. L., Biedenbach, D. J., Jones, R. N., Rhomberg, P. R., and Mutnick, A. H. (2002). Antimicrobial susceptibility patterns of beta-hemolytic and viridans group streptococci: report from the SENTRY Antimicrobial Surveillance Program (1997-2000). Diagn. Microbiol. Infect. Dis. 43, 157-162. doi: 10.1016/S0732-8893(02)00374-7
Han, X. Y., Kamana, M., and Rolston, K. V. I. (2006). Viridans streptococci isolated by culture from blood of cancer patients: clinical and microbiologic analysis of 50 cases. J. Clin. Microbiol. 44, 160-165. doi: 10.1128/JCM.44.1.160165.2006

Hiramatsu, K., Katayama, Y., Matsuo, M., Sasaki, T., Morimoto, Y., Sekiguchi, A., et al. (2014). Multi-drug-resistant Staphylococcus aureus and future chemotherapy. J. Infect. Chemother. 20, 593-601. doi: 10.1016/j.jiac.2014.08.001

Høiby, N., Bjarnsholt, T., Givskov, M., Molin, S., and Ciofu, O. (2010). Antibiotic resistance of bacterial biofilms. Int. J. Antimicrob. Agents 35, 322-332. doi: 10.1016/j.ijantimicag.2009.12.011

Jahanfar, S., Ng, C. J., and Teng, C. L. (2013). Antibiotics for mastitis in breastfeeding women. Cochrane Database Syst. Rev. 2, CD005458. doi: 10.1002/ 14651858.CD005458.pub3

Joffe, T. H., and Simpson, N. A. (2009). Cesarean section and risk of asthma. The role of intrapartum antibiotics: a missing piece? J. Pediatr. 154, 154. doi: 10.1016/j.jpeds.2008.08.039

Kärpänoja, P., Harju, I., Rantakokko-Jalava, K., Haanperä, M., and Sarkkinen, H. (2014). Evaluation of two matrix-assisted laser desorption ionization-time of flight mass spectrometry systems for identification of viridans group streptococci. Eur. J. Clin. Microbiol. Infect. Dis. 33, 779-788. doi: 10.1007/ s10096-013-2012-8

Kristich, C. J., Rice, L. B., and Arias, C. A. (2014). "Enterococcal InfectionTreatment and Antibiotic Resistance," in Enterococci: From Commensals to Leading Causes of Drug Resistant Infection, eds M. S. Gilmore, D. B. Clewell, Y. Ike, and N. Shankar (Boston: Massachusetts Eye and Ear Infirmary). Available at: http://www.ncbi.nlm.nih.gov/books/NBK190420/ [accessed June 27, 2017].

Lawrence, R. A., and Lawrence, R. M. (2011). Breastfeeding: A Guide for the Medical Professional - Expert Consult. Amsterdam: Elsevier Health Sciences.

Magiorakos, A.-P., Srinivasan, A., Carey, R. B., Carmeli, Y., Falagas, M. E., Giske, C. G., et al. (2012). Multidrug-resistant, extensively drug-resistant and pandrug-resistant bacteria: an international expert proposal for interim standard definitions for acquired resistance. Clin. Microbiol. Infect. 18, 268-281. doi: 10.1111/j.1469-0691.2011.03570.x

Martín, V., Mediano, P., Campo, R., del Rodríguez, J. M., and Marín, M. (2016). Streptococcal diversity of human milk and comparison of different methods for the taxonomic identification of streptococci. J. Hum. Lact. 32, N84-N94. doi: 10.1177/0890334415597901

Mathelin, C., Riegel, P., Chenard, M.-P., and Brettes, J.-P. (2005). Association of corynebacteria with granulomatous mastitis. Eur. J. Obstet. Gynecol. Reprod. Biol. 119, 260-261. doi: 10.1016/j.ejogrb.2004.08.003

Mazzola, G., Murphy, K., Ross, R. P., Di Gioia, D., Biavati, B., Corvaglia, L. T., et al. (2016). Early gut microbiota perturbations following intrapartum antibiotic prophylaxis to prevent group B streptococcal disease. PLoS ONE 11:e0157527. doi: 10.1371/journal.pone.0157527

Mediano, P., Fernández, L., Jiménez, E., Arroyo, R., Espinosa-Martos, I., Rodríguez, J. M., et al. (2017). Microbial diversity in milk of women with mastitis: potential role of coagulase-negative staphylococci, viridans group streptococci, and corynebacteria. J. Hum. Lact. 33, 309-318. doi: 10.1177/ 0890334417692968

Mediano, P., Fernández, L., Rodríguez, J. M., and Marín, M. (2014). Case-control study of risk factors for infectious mastitis in Spanish breastfeeding women. BMC Pregnancy Childbirth 14:195. doi: 10.1186/1471-2393-14-195

Murk, W., Risnes, K. R., and Bracken, M. B. (2011). Prenatal or early-life exposure to antibiotics and risk of childhood asthma: a systematic review. Pediatrics 127 , 1125-1138. doi: 10.1542/peds.2010-2092

Nam, H. M., Lim, S. K., Moon, J. S., Kang, H. M., Kim, J. M., Jang, K. C., et al. (2010). Antimicrobial resistance of enterococci isolated from mastitic bovine milk samples in Korea. Zoonoses Public Health 57, e59-e64. doi: 10.1111/j.18632378.2009.01307.x

Nomura, F. (2015). Proteome-based bacterial identification using matrix-assisted laser desorption ionization-time of flight mass spectrometry (MALDI-TOF MS): A revolutionary shift in clinical diagnostic microbiology. Biochim. Biophys. Acta 1854, 528-537. doi: 10.1016/j.bbapap.2014.10.022

Olender, A. (2013). Antibiotic resistance and detection of the most common mechanism of resistance (MLSB) of opportunistic Corynebacterium. Chemotherapy 59, 294-306. doi: 10.1159/000357467

Osuka, H., Nakajima, J., Oishi, T., Funayama, Y., Ebihara, T., Ishikawa, H., et al. (2016). High-level aminoglycoside resistance in Enterococcus faecalis and 
Enterococcus faecium causing invasive infection: Twelve-year surveillance in the Minami Ibaraki Area. J. Infect. Chemother. 22, 61-63. doi: 10.1016/j.jiac.2015. 09.003

Otto, M. (2009). Staphylococcus epidermidis-the "accidental" pathogen. Nat. Rev. 7, 555-567. doi: 10.1038/nrmicro2182

Ramanan, P., Barreto, J. N., Osmon, D. R., and Tosh, P. K. (2014). Rothia bacteremia: a 10-year experience at Mayo Clinic, Rochester, Minnesota. J. Clin. Microbiol. 52, 3184-3189. doi: 10.1128/JCM.01270-14

Reddy, P., Qi, C., Zembower, T., Noskin, G. A., and Bolon, M. (2007). Postpartum mastitis and community-acquired methicillin-resistant Staphylococcus aureus. Emerg. Infect. Dis. 13, 298-301. doi: 10.3201/eid1302.060989

Rutten, N. B. M. M., Rijkers, G. T., Meijssen, C. B., Crijns, C. E., Oudshoorn, J. H., van der Ent, C. K., et al. (2015). Intestinal microbiota composition after antibiotic treatment in early life: the INCA study. BMC Pediatr. 15, 204. doi: 10.1186/s12887-015-0519-0

Sahal, G., and Bilkay, I. S. (2014). Multi drug resistance in strong biofilm forming clinical isolates of Staphylococcus epidermidis. Braz. J. Microbiol. 45, 539-544. doi: 10.1590/S1517-83822014005000042

Schmidt, T., Kock, M. M., and Ehlers, M. M. (2015). Diversity and antimicrobial susceptibility profiling of staphylococci isolated from bovine mastitis cases and close human contacts. J. Dairy Sci. 98, 6256-6269. doi: 10.3168/jds.2015-9715

Scott, J. A., Robertson, M., Fitzpatrick, J., Knight, C., and Mulholland, S. (2008). Occurrence of lactational mastitis and medical management: A prospective cohort study in Glasgow. Int. Breastfeed. J. 3:21. doi: 10.1186/1746-435 8-3-21

Singhal, N., Kumar, M., Kanaujia, P. K., and Virdi, J. S. (2015). MALDI-TOF mass spectrometry: an emerging technology for microbial identification and diagnosis. Front. Microbiol. 6:791. doi: 10.3389/fmicb.2015.00791

Sood, S., Malhotra, M., Das, B. K., and Kapil, A. (2008). Enterococcal infections \& antimicrobial resistance. Indian J. Med. Res. 128, 111-121.

Soto, A., Martin, V., Jimenez, E., Mader, I., Rodriguez, J. M., and Fernandez, L. (2014). Lactobacilli and Bifidobacteria in human breast milk: influence of antibiotherapy and other host and clinical factors. J. Pediatr. Gastroenterol. Nutr. 59, 78-88. doi: 10.1097/MPG.0000000000000347

Stensballe, L. G., Simonsen, J., Jensen, S. M., Bønnelykke, K., and Bisgaard, H. (2013). Use of antibiotics during pregnancy increases the risk of asthma in early childhood. J. Pediatr 162, 832.e-838.e. doi: 10.1016/j.jpeds.2012.09.049

Szczuka, E., Makowska, N., Bosacka, K., Słotwiñska, A., and Kaznowski, A. (2016). Molecular basis of resistance to macrolides, lincosamides and streptogramins in Staphylococcus hominis. Folia Microbiol. 61, 143-147. doi: 10.1007/s12223-0150419-6

Taponen, S., Nykäsenoja, S., Pohjanvirta, T., Pitkälä, A., and Pyörälä, S. (2016). Species distribution and in vitro antimicrobial susceptibility of coagulase-negative staphylococci isolated from bovine mastitic milk. Acta Vet. Scand. 58, 12. doi: 10.1186/s13028-016-0193-8

Taylor, G. B., Paviour, S. D., Musaad, S., Jones, W. O., and Holland, D. J. (2003). A clinicopathological review of 34 cases of inflammatory breast disease showing an association between corynebacteria infection and granulomatous mastitis. Pathology 35, 109-119. doi: 10.1080/0031302031000082197

Tomazi, T., Gonçalves, J. L., Barreiro, J. R., de Campos Braga, P. A., Prada e Silva, L. F., Eberlin, M. N., et al. (2014). Identification of coagulase-negative staphylococci from bovine intramammary infection by matrix-assisted laser desorption ionization-time of flight mass spectrometry. J. Clin. Microbiol 52, 1658-1663. doi: 10.1128/JCM.03032-13

U.S. Department of Health and Human Services (2011). The Surgeon General's Call to Action to Support Breastfeeding. Rockville, MD: Office of the Surgeon General (US). Available at: http://www.ncbi.nlm.nih.gov/books/NBK52682/ [accessed June 27, 2017].

Wendlandt, S., Feßler, A. T., Monecke, S., Ehricht, R., Schwarz, S., and Kadlec, K. (2013). The diversity of antimicrobial resistance genes among staphylococci of animal origin. Int. J. Med. Microbiol. 303, 338-349. doi: 10.1016/j.ijmm.2013. 02.006

WHO (2000). Mastitis: Causes and Management. Geneva: Department of Child and Adolescent Health and Development. Available at: http://www.who.int/maternal_child_adolescent/documents/fch_cah_00_13/en/ [accessed June 27, 2017].

Willing, B. P., Russell, S. L., and Finlay, B. B. (2011). Shifting the balance: antibiotic effects on host-microbiota mutualism. Nat. Rev. Microbiol. 9, 233-243. doi: $10.1038 /$ nrmicro2536

Winstanley, T., and Courvalin, P. (2011). Expert systems in clinical microbiology. Clin. Microbiol. Rev. 24, 515-556. doi: 10.1128/CMR.00061-10

Witt, A. M., Burgess, K., Hawn, T. R., and Zyzanski, S. (2014). Role of oral antibiotics in treatment of breastfeeding women with chronic breast pain who fail conservative therapy. Breastfeed. Med. 9, 63-72. doi: 10.1089/bfm.2013.0093

Conflict of Interest Statement: The authors declare that the research was conducted in the absence of any commercial or financial relationships that could be construed as a potential conflict of interest.

Copyright (®) 2017 Marín, Arroyo, Espinosa-Martos, Fernández and Rodríguez. This is an open-access article distributed under the terms of the Creative Commons Attribution License (CC BY). The use, distribution or reproduction in other forums is permitted, provided the original author(s) or licensor are credited and that the original publication in this journal is cited, in accordance with accepted academic practice. No use, distribution or reproduction is permitted which does not comply with these terms. 